\section{MS13-P14 Using X-ray crystallography to validate the computational design of enantioselective epoxide hydrolases}

Marco Dal Lago ${ }^{1}$, Hein J. Wijma ${ }^{2}$, Anke C. Terwisscha van Scheltinga ${ }^{1}$, Dick B. Janssen ${ }^{2}$, Andy-Mark W.H. Thunnissen

1. Laboratory of Biophysical Chemistry, Groningen Biomolecular Sciences and Biotechnology Institute, University of Groningen, Nijenborgh 7, 9747AG Groningen, The Netherlands

2. Biotransformation and Biocatalysis, Groningen Biomolecular Science and Biotechnology Institute, University of Groningen, Nijenborgh 4, 9747AG, Groningen, the Netherlands

email: m.dal.lago@rug.nl

A computational design strategy to engineer complementary enantioselective epoxide hydrolase variants for the production of highly enantioenriched (S,S)-diols and (R,R)-diols from meso-epoxides has been recently developed. Key features of this strategy (CASCO, catalytic selectivity by computational design) are the use of RosettaDesign to design mutations that favor binding of the substrate in a predefined orientation, the introduction of steric hindrance to prevent unwanted substrate binding modes, and ranking of designs by high-throughput molecular dynamics simulations. Predicting the correct conformation of enzyme variants carrying multiple mutations is a critical step of the CASCO procedure. Validation of the computational methods requires experimental determination of structures by X-ray crystallography.

Here we present high resolution X-ray structures of 5 multi-site mutants of limonene epoxide hydrolase (LEH-pro-RR3, LEH-pro-RR7, LEH-pro-RR8, LEH-pro-SS3 and LEH-pro-SS16) designed with CASCO. We solved structures of mutants showing opposite enantioselectivity, mutants generated with different steric hindrance restraints, as well as mutants showing a different enantioselectivity from the predicted one. We compare the experimentally determined structures and computational predictions, focusing our attention on evaluating the accuracy of the mutant structures generated by RosettaDesign as well as on the possibility to correct a wrong prediction by MD simulations. Most predicted structures and averaged structures of MD simulations were in excellent agreement with the crystal structures. Some deviations between the Rosetta structural predictions and the X-ray structures concern the side chain conformation of mutated amino acids or their neighboring residues. The conformations generated by RosettaDesign were maintained during the MD simulations. From the comparative analysis we conclude that apart from a few exceptions the computational models overall resemble the crystal structures. The results indicate that the discrepancy different predicted and measured enantioselectivity of the LEH-pro-RR3 mutant was not caused by erroneous modelling of the designed enzyme, but by incorrect prediction of its enantioselectivity. Therefore, more accurate in silico predictions of catalytic selectivity will further improve the success rate of the computational protocol for designing enantioselective biocatalysts.

Keywords: protein crystallography, protein engineering, CASCO, epoxide hydrolase
MS14 Biomineralogical

crystallography and bioinspired inorganic materials

Chairs: Wolfgang Schmahl, Fernandez-Diaz Lurdes

MS14-P1 Synergistic antimicrobial effect of silver and other metals in bimetallic complexes

Paula C. Corcosa ${ }^{1}$, Aurélien Crochet $^{1}$, Katharina M. Fromm ${ }^{1}$

1. Department of Chemistry, University of Fribourg, Chemin du Musée 9, 1700 Fribourg, Switzerland

email: paula.corcosa@unifr.ch

The precious metal silver is aging for its excellent antimicrobial properties as throwing silver coins in fountains is not only a lovely old tradition. ${ }^{[1]}$ It has been recognized to play an important role concerning medical applications, for example the coating of implants with $\mathrm{Ag}^{0}$ or $\mathrm{Ag}^{\mathrm{I}}$ coordination compounds to avoid infection due to bacterial biofilms formation. ${ }^{[2]}$

The research of the FROMM group with respect to antimicrobial silver compounds was focused on silver coordination networks, meaning short PEG oligomers functionalized with (iso-)nicotinic acid as ligands.

Thus, the aim of the recent project is to create new Ag complexes with bioinspired ligands, for example pyridylalanine or picolinic acid as well as with derivatives of phenylalanine or aminobenzoic acid. Furthermore, we try to synthesize bimetallic complexes combining silver and another metal such as $\mathrm{Zn}^{\mathrm{II}}$ or $\mathrm{Cu}^{\mathrm{II}}$ with the ambition to generate synergistic antimicrobial effects and to elucidate structural characteristics.

${ }^{[1]}$ Fromm, K. M. Nat. Chem. 2011, 3, 178.

${ }^{[2]}$ Vig Slenters, T. et al. Materials 2010, 3, 3407-3429.

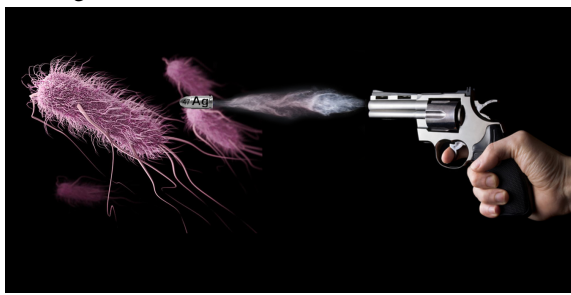

Figure 1. Silver fights against bacteria

Keywords: Bimetallic complexes, Silver, Bioinspired ligands 